\title{
AN ASSESSMENT OF THE PERFORMANCE OF THE OPERATIONAL GLOBAL ENSEMBLE FORECAST SYSTEMS IN PREDICTING THE FORECAST UNCERTAINTY
}

\author{
A Thesis \\ by \\ CARLEE FRANCES LOESER \\ Submitted to the Office of Graduate and Professional Studies of \\ Texas A\&M University \\ in partial fulfillment of the requirement for the degree of \\ MASTER OF SCIENCE
}

\begin{abstract}
Chair of Committee, Istvan Szunyogh
Committee Members, Robert Korty

Robert Hetland

Head of Department, Ping Yang
\end{abstract}

August 2016

Major Subject: Atmospheric Sciences

Copyright 2016 Carlee Frances Loeser 


\begin{abstract}
This study investigates the efficiency of the operational global ensemble forecast systems in capturing the spatiotemporal evolution of the forecast uncertainty. It has two novel aspects: first, it extends the results of an earlier study from 2012 to 2015; second, it documents the first attempts to predict the reliability of the ensembles in capturing the uncertain forecast features and the 95th percentile value of the forecast error for operational ensembles. It is found that the main characteristics of the systems of the different centers in their efficiency in representing the spatiotemporal evolution of the forecast uncertainty have not changed much in the last three years. The only exception is the UKMO ensemble, whose performance improved in predicting the total magnitude of the uncertainty, but greatly degraded in predicting the patterns of forecast uncertainty. All ensembles were found to have major difficulties with predicting the large scale atmospheric flow in the forecast range longer than 10 days. These difficulties are due to the inability of the models to maintain the large-scale zonal anomalies of the atmospheric flow in the long forecast range. It was also found that the flow-dependent reliability of the ensembles in capturing the local structure of the forecast uncertainty and the 95th percentile value of the forecast error can accurately be predicted.
\end{abstract}




\section{ACKNOWLEDGEMENTS}

I would like to thank my advisor, Dr. Istvan Szunyogh, as well as my committee, Dr. Robert Korty and Dr. Robert Hetland. Special thanks to Michael Herrera, as he helped me every step of the way with graduate school and with this thesis project. I could not have finished graduate school without his help and support.

I would also like to thank my family and friends, for always supporting me and pushing me to do my best. Special thanks to the lifelong friends I made in my graduate class.

I dedicate this thesis to my sweet grandparents; I miss you both everyday. 


\section{TABLE OF CONTENTS}

Page

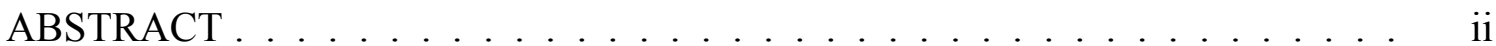

ACKNOWLEDGEMENTS ...................... . . iii

TABLE OF CONTENTS ....................... . . iv

LIST OF FIGURES . . . . . . . . . . . . . . . . . . . . . vi

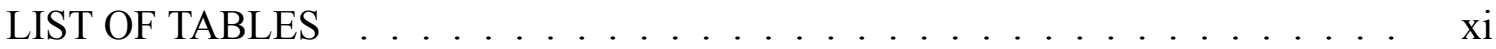

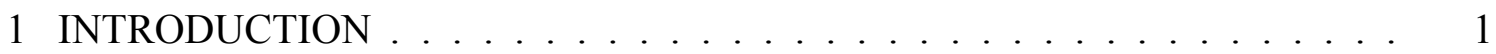

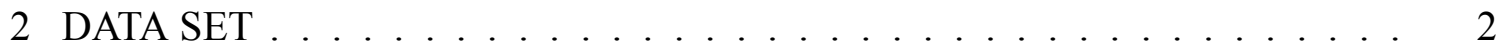

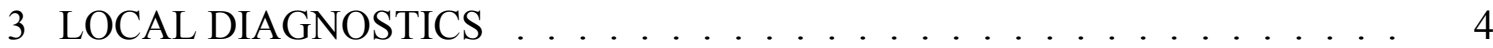

3.1 Local state vectors . . . . . . . . . . . . . . . . . . . . . . 4

3.2 Local ensemble perturbations . . . . . . . . . . . . . . 5

3.3 Diagnostics ........................... 6

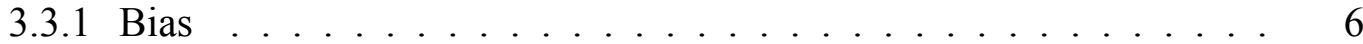

3.3 .2 Variance ........................ 8

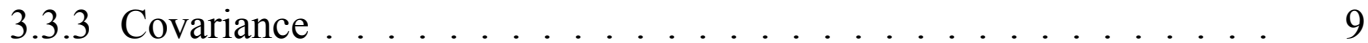

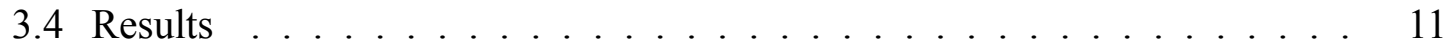

3.4.1 Comparison of $V S, T V$, and $T V S \ldots \ldots \ldots \ldots$

3.4 .2 The evolution of $M^{2} \ldots \ldots \ldots 13$

4 EVALUATION OF THE PREDICTIVE SCHEMES . . . . . . . . . . . 16

4.1 The predictive linear relations f . . . . . . . . . . . . . . . 16

4.1.1 The lower bound of the explained variance . . . . . . . . . . . 16

4.1.2 The 95th percentile value of the forecast error . . . . . . . . . . 17

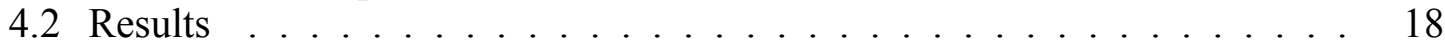

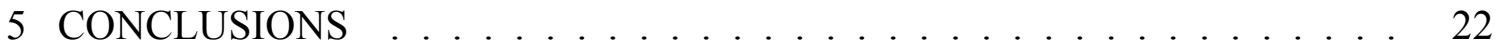

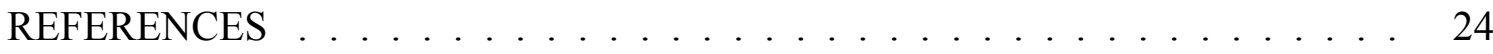




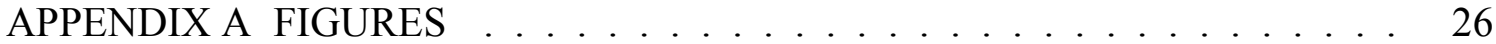

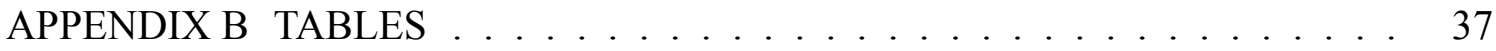




\section{LIST OF FIGURES}

FIGURE

Page

A.1 Spatiotemporal evolution of the diagnostics, $T V, V S, T V S$, and $M^{2}$, for the ECMWF (left) and NCEP (right) ensembles, averaged over the Northern Hemisphere extratropics and all of the available forecasts. . . 26

A.2 Spatiotemporal evolution of the diagnostics, $T V, V S, T V S$, and $M^{2}$, for the CMC (top left), JMA (top right), KMA (bottom left), and UKMO (bottom right) ensembles, averaged over the Northern Hemisphere extratropics and all of the available forecasts. The maximum number of lead times vary for these models. . . . . . . . . . . . .

A.3 Spatial distribution of mean bias for the ECMWF ensemble in $(J / k g)^{1 / 2}$, averaged over all of the forecasts, for analysis (top left), the 72-hour (top right), the 120-hour (bottom left), and the 360-hour (bottom right) forecast lead times, shown as color shades. The black contours represent the mean geopotential height at $500 \mathrm{hPa}$. The heavy dashed line denotes the southern boundary of the verification region $\left(30^{\circ} \mathrm{N}\right) . \ldots 28$ 
A.4 Spaghetti diagram for the ECMWF ensemble at analysis time (top left), the 72-hour (top right), the 120-hour (bottom left), and the 360-hour (bottom right) forecast lead times, represented by the ensemble members for the averaged forecasts over the time period, in gray, and the mean ECMWF analyses as the verification, in black. The ensemble members and verification are represented as $5625 \mathrm{gpm}$ isohypses. . . . . . . .

A.5 Spaghetti diagram for the ECMWF (top), NCEP (middle), and CMC (bottom) ensembles at the 360-hour forecast lead time, represented by the ensemble members for the averaged forecasts over the time period, in gray, and the mean ECMWF analyses as the verification, in black. The ensemble members and verification are represented as $5625 \mathrm{gpm}$ isohypses. (Results are not shown for the remaining ensembles, because they do not provide forecasts at the 360 -hour lead time.) $\ldots \ldots \ldots$

A.6 Zonal anomalies of the time-mean flow for January-February for the ECMWF ensemble from 2012 (left) and from 2015 (right). The color shades represent the zonal anomalies (in geopotential meters) and the black contours represent the mean geopotential height at $500 \mathrm{hPa}$. The heavy dashed line denotes the southern boundary of the verification re-

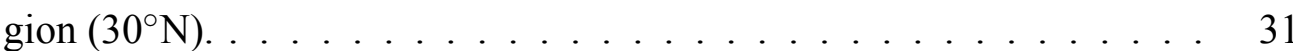


A.7 Spatial distribution of explained variance for the ECMWF ensemble, averaged over all of the forecasts, for analysis (top left), the 72-hour (top right), the 120-hour (bottom left), and 360-hour (bottom right) forecast lead times, shown as the color shades. The black contours represent the mean geopotential height at $500 \mathrm{hPa}$. The heavy dashed line denotes the southern boundary of the verification region $\left(30^{\circ} \mathrm{N}\right), \ldots \ldots 32$

A.8 The joint probability distribution of the E-dimension and the explained variance for the ECMWF ensemble in the Northern Hemisphere extratropics for the analysis (top left), the 72-hour (top right), the 120-hour (bottom left), and the 360-hour (bottom right) forecast lead times. The bin increments are defined as $\Delta E=0.25$ and $\Delta e v=0.005$. The maximum possible value for E-dimension for the ECMWF ensemble is $50 \ldots \ldots 33$ 
A.9 Mean E-dimension and the minimum of explained variance of data divided equally into 100 bins for the $\mathrm{NH}$ extratropics. The training data is represented as triangles, and the linear regression line is fitted to these data. The test data is represented as open circles, and would fall on this line if the linear model was perfect. Shown are the distributions for the analysis time (top left), the 72-hour (top right), the 120-hour (bottom left), and the 360-hour (bottom right) forecast lead times. The legends show the average correlation values between mean E-dimension and minimum explained variance for the training data set $\left(R^{2}\right.$ training $)$ and test data set ( $R^{2}$ test), as well as the average $a$ and $b$ values. . . . .

A.10 Mean E-dimension and the 5th percentile of explained variance of data divided equally into 100 bins for the $\mathrm{NH}$ extratropics. The training data is represented as triangles, and the linear regression line is fitted to these data. The test data is represented as open circles, and would fall on this line if the linear model was perfect. Shown are the distributions for the analysis time (top left), the 72-hour (top right), the 120-hour (bottom left), and the 360-hour (bottom right) forecast lead times. The legends show the average correlation values between mean E-dimension and 5th percentile of explained variance for the training data set $\left(R^{2}\right.$ training) and test data set $\left(R^{2}\right.$ test), as well as the average $a$ and $b$ values. . . . . . . 35 
A.11 Mean $v s_{\ell}^{1 / 2}$ and the 95th percentile of $t v_{\ell}^{1 / 2}$ of data divided equally into 100 bins for the $\mathrm{NH}$ extratropics. The training data is represented as triangles, and the linear regression line is fitted to these data. The test data is represented as open circles, and would fall on this line if the linear model was perfect. Shown are the distributions for the analysis time (top left), the 72-hour (top right), the 120-hour (bottom left), and the 360 hour (bottom right) forecast lead times. The legends show the average correlation values between mean $v s_{\ell}^{1 / 2}$ and the 95th percentile of $t v_{\ell}^{1 / 2}$ for the training data set ( $R^{2}$ training) and test data set $\left(R^{2}\right.$ test), as well as the average $c$ and $d$ values. . . . . . . . . . . . . . . . 36 


\section{LIST OF TABLES}

TABLE

Page

B.1 Ensemble Forecast Systems . . . . . . . . . . . . . . . . . 37

B.2 $R^{2}$ Values for the regressions of Sec. $4 \ldots \ldots 38$ 


\section{INTRODUCTION}

This study investigates the efficiency of the global forecast ensembles in capturing the spatiotemporal evolution of the forecast uncertainty. It is an extension of the research efforts that started with an analysis of data generated by a research forecast system that was based on the model component of the Global Forecast System (GFS) of the National Centers for Environmental Prediction (NCEP) (Kuhl et al., 2007; Satterfield and Szunyogh, 2010, 2011). In a follow up paper, Herrera et al. (2016) applied the diagnostics of the earlier papers to global ensemble forecast data from the world's leading operational numerical weather prediction centers for January-February 2012.

The present study has two novel aspects. First, it extends the investigations of Herrera et al. (2016) to data from January-February 2015, allowing for an assessment of the 3-year progress made by the prediction centers between 2012 and 2015. Second, and perhaps more importantly, it documents our first attempt to validate two prognostic relations found by Satterfield and Szunyogh $(2010,2011)$. One of these relations provides a tool for the routine prediction of the reliability of the ensemble in capturing the uncertain forecast features. The other is for the prediction of the 95 th percentile value of the forecast error. In what follows, we describe the operational ensemble data that we analyze (section 2), explain and apply the local diagnostics adapted from Herrera et al. (2016) (section 3), evaluate the performance of the predictive schemes of Satterfield and Szunyogh $(2010,2011)$ for the ECMWF ensemble (section 4), and offer some conclusions (section 5). 


\section{DATA SET}

We analyze data provided by the forecast centers through the THORPEX Interactive Grand Global Ensemble (TIGGE). TIGGE includes operational global model forecasts from 10 major numerical weather prediction centers (Bougeault and Coauthors, 2010; Swinbank and Coauthors, 2016). We process forecast data from the

- European Centre for Medium-Range Weather Forecasts (ECMWF)

- US National Centers for Environmental Prediction (NCEP)

- UK Met Office (UKMO)

- Japan Meteorological Agency (JMA)

- Korean Meteorological Administration (KMA)

- Meteorological Service of Canada (CMC)

Data from the remaining four centers, China Meteorological Administration (CMA), Météo-France, the Australian Bureau of Meteorology (BoM), and Centro de Previsão de Tempo e Estudos Climáticos (CPTEC), were unavailable, or had quality issues for the investigated time period of January-February 2015. Table B.1 summarizes the main features of the ensembles of the different centers. Herrera et al. (2016) provided a more detailed overview of the ensemble generation techniques of the different centers. In what follows, we give only a brief summary of the most important changes made to those techniques between 2012 and 2015.

Since 2012, KMA has moved from using bred vector initial condition perturbations 
to using perturbations generated by an Ensemble Transform Kalman Filter (ETKF). They now also use a combination of Stochastic Kinetic Energy Backscatter (SKEB) and Random Parameters (RP) schemes to simulate the effects of random model errors. UKMO also switched to a combination of SKEB and RP schemes, reduced the number of ensemble members to 11 from 14 members, and shortened the maximum forecast time from 360 to 168 hours. JMA has increased the frequency of ensemble forecasts from once daily to twice daily, and extended the maximum forecast time from 216 to 264 hours. 


\section{LOCAL DIAGNOSTICS}

A forecast ensemble samples the flow-dependent multivariate probability distribution of the present and future atmospheric states given the sources of forecast uncertainty. We verify the ensemble-based predictions of the first and second statistical moments (mean, variances, and covariances) of the probability distribution of the atmospheric states.

\subsection{Local state vectors}

Let $\ell$ be the index that identifies the horizontal location of a grid point. At location $\ell$, we define the components of the local state vector $\mathbf{x}_{\ell}$ by the two horizontal wind and the temperature grid point variables in a rectangular box $V_{\ell}$ centered at $\ell$, in the two horizontal directions. The wind and temperature components of $\mathbf{x}_{\ell}$ are scaled such that the Euclidean norm of the local state vector has dimension of square-root of energy (for the definition of the scaling factors see Talagrand, 1981; Buizza et al., 1993; Oczkowski et al., 2005). Following Satterfield and Szunyogh (2010, 2011); Herrera et al. (2016), we choose $V_{\ell}$ to be $12.5^{\circ}$-by- $12.5^{\circ}$ horizontally and extend from $1000 \mathrm{hPa}$ to $200 \mathrm{hPa}$ vertically. (In the midlatitudes, the horizontal dimension of $V_{\ell}$ is about $1000 \mathrm{~km}$ by $1000 \mathrm{~km}$.) We choose a horizontal dimension of this size because we focus on error growth at synoptic scales. A horizontal resolution of $2.5^{\circ}$-by- $2.5^{\circ}$ and 8 pressure levels between $1000 \mathrm{hPa}$ and $200 \mathrm{hPa}$ are chosen from the TIGGE archive, so the dimension of the local state vector is $3 \times 5 \times$ $5 \times 8=600$. 
The local state vector $\mathbf{x}_{\ell}^{t}$ that represents the projection of the true state on the grid can be decomposed as

$$
\mathbf{x}_{\ell}^{t}=\overline{\mathbf{x}}_{\ell}^{t}+\boldsymbol{\varepsilon}_{\ell}^{r}
$$

where $\overline{\mathbf{x}}_{\ell}^{t}$ represents the (unknown) true mean of the probability distribution of the local state given all sources of forecast uncertainty, and $\boldsymbol{\varepsilon}_{\ell}^{r}$ is the random variable that represents the forecast uncertainty. Notice that the mean of $\boldsymbol{\varepsilon}_{\ell}^{r}$ is $\mathbf{0}$. The ultimate goal of ensemble prediction, stated here in the context of local state vectors, is to predict $\overline{\mathbf{x}}_{\ell}^{t}$ and the probability distribution of $\boldsymbol{\varepsilon}_{\ell}^{r}$. In this paper, our focus is on the predictions of $\overline{\mathbf{x}}_{\ell}^{t}$ and the second statistical moments (variances and covariances) of $\boldsymbol{\varepsilon}_{\ell}^{r}$.

\subsection{Local ensemble perturbations}

For a $K$-member ensemble of local state vectors $\mathbf{x}_{\ell}^{k}, k=1,2, \ldots, K$, the ensemble of local ensemble perturbations, $\mathbf{X}_{\ell}^{k}, k=1,2, \ldots, K$, is defined by

$$
\mathbf{X}_{\ell}^{k}=\mathbf{x}_{\ell}^{k}-\overline{\mathbf{x}}_{\ell}
$$

where $\overline{\mathbf{x}}_{\ell}$ is the local ensemble mean

$$
\overline{\mathbf{x}}_{\ell}=\frac{1}{K} \sum_{k=1}^{K} \mathbf{x}_{\ell}^{k} .
$$

The ensemble based prediction of the variances and covariances of the forecast uncertainty are described by the local ensemble covariance matrix

$$
\mathbf{P}_{\ell}=\frac{1}{K-1} \sum_{k=1}^{K} \mathbf{X}_{\ell}^{k}\left[\mathbf{X}_{\ell}^{k}\right]^{T}
$$


where the local ensemble perturbations are represented by column vectors, and the superscript $T$ denotes the matrix transpose. The ensemble mean $\overline{\mathbf{x}}_{\ell}$ is the ensemble based prediction of $\overline{\mathbf{x}}_{\ell}^{t}$, while $\mathbf{P}_{\ell}$ is the prediction of the covariance matrix of $\boldsymbol{\varepsilon}_{\ell}^{r}$.

\subsection{Diagnostics}

Because the true state $\mathbf{x}_{\ell}^{t}$ is unknown, forecasts are always verified against a proxy $\mathbf{x}_{\ell}^{p}$ for the true state. In our diagnostic calculations, $\mathbf{x}_{\ell}^{p}$ is defined by the appropriate grid point values of the operational ECMWF control analyses. For the verification of the ECMWF model forecasts, the operational NCEP control analyses are used as the proxy $\mathbf{x}_{\ell}^{p}$. We assume that the error in the proxy can be described by the random variable $\boldsymbol{\varepsilon}_{\ell}^{p}$, that is,

$$
\mathbf{x}_{\ell}^{p}=\mathbf{x}_{\ell}^{t}+\boldsymbol{\varepsilon}_{\ell}^{p}
$$

\subsubsection{Bias}

The relationship

$$
\overline{\mathbf{x}}_{\ell}=\overline{\mathbf{x}}_{\ell}^{t}
$$

cannot be verified for a single ensemble forecast and location, because an accurate independent estimate of $\overline{\mathbf{x}}_{\ell}^{t}$ is not available in a realistic situation. In other words, no practical technique exists to quantify the error in a prediction of the spatiotemporally evolving mean of the probability distribution of the state. A verifiable, necessary, condition for Eq. (3.6) to hold is

$$
E\left[\overline{\mathbf{x}}_{\ell}\right]=E\left[\overline{\mathbf{x}}_{\ell}^{t}\right]
$$


where $E[\cdot]$ is the expected value for forecasts started at different times. Under the standard hypothesis of ensemble forecasting that the processes that govern the evolution of $\mathbf{x}_{\ell}$ are ergodic, Eq. (3.7) can be written as

$$
E\left[\overline{\mathbf{x}}_{\ell}\right]=E\left[\mathbf{x}_{\ell}^{t}\right]
$$

Making the additional assumption that $\mathbf{x}_{\ell}^{p}$ is an unbiased estimate of $\mathbf{x}_{\ell}^{t}\left(E\left[\boldsymbol{\varepsilon}_{\ell}^{p}\right]=\mathbf{0}\right)$, substituting for $\mathbf{x}_{\ell}^{t}$ in Eq. (3.8) from Eq. (3.5), and rearranging the resulting equation leads to

$$
E\left[\mathbf{x}_{\ell}^{p}-\overline{\mathbf{x}}_{\ell}\right]=\mathbf{0}
$$

Introducing the notation

$$
\delta \mathbf{x}_{\ell}=\mathbf{x}_{\ell}^{p}-\overline{\mathbf{x}}_{\ell}
$$

Eq. (3.9) can be written in the equivalent form

$$
E\left[\delta \mathbf{x}_{\ell}\right]=\mathbf{0} .
$$

The mean $M_{\ell}=E\left[\delta \mathbf{x}_{\ell}\right]$ for location $\ell$ can be estimated by computing the average of a sample of $\delta \mathbf{x}_{\ell}$ for a sufficiently long verification time period. The result is a map of the systematic error (bias) of the ensemble mean forecasts. The values of $M_{\ell}$ on the map can be averaged over the locations $\ell$ to obtain a single number, which we will denote by $M$, for the characterization of the bias. 


\subsubsection{Variance}

Under the assumption that Eq. (3.6) is satisfied, the trace of $\mathbf{P}_{\ell}, v s_{\ell}$, is a prediction of the variance of $\boldsymbol{\varepsilon}_{\ell}^{r}$. That is, ideally, the relation

$$
v s_{\ell}=\overline{\left(\boldsymbol{\varepsilon}_{\ell}^{r}\right)^{T} \boldsymbol{\varepsilon}_{\ell}^{r}}=\overline{\left|\boldsymbol{\varepsilon}_{\ell}^{r}\right|^{2}}
$$

would be satisfied for each forecast and location. Similar to the situation for the mean, this condition cannot be verified for a single ensemble forecast and location. Taking the expected value of Eq. (3.12) and making use of the ergodic hypothesis lead to

$$
E\left[v s_{\ell}\right]=E\left[\left(\boldsymbol{\varepsilon}_{\ell}^{r}\right)^{T} \boldsymbol{\varepsilon}_{\ell}^{r}\right]=E\left[\left|\boldsymbol{\varepsilon}_{\ell}^{r}\right|^{2}\right] .
$$

Under the assumption that the ensemble satisfies Eq. (3.6),

$$
\begin{aligned}
\boldsymbol{\varepsilon}_{\ell}^{r} & =\mathbf{x}_{\ell}^{t}-\overline{\mathbf{x}}_{\ell}^{t} \\
& =\mathbf{x}_{\ell}^{p}-\boldsymbol{\varepsilon}_{\ell}^{p}-\overline{\mathbf{x}}_{\ell}^{t} \\
& =\mathbf{x}_{\ell}^{p}-\boldsymbol{\varepsilon}_{\ell}^{p}-\overline{\mathbf{x}}_{\ell} \\
& =\delta \mathbf{x}_{\ell}-\boldsymbol{\varepsilon}_{\ell}^{p}
\end{aligned}
$$

and the right-hand side of Eq. (3.13) can be expanded as

$$
\begin{aligned}
E\left[\left(\boldsymbol{\varepsilon}_{\ell}^{r}\right)^{T} \boldsymbol{\varepsilon}_{\ell}^{r}\right] & =E\left[\left(\delta \mathbf{x}_{\ell}-\boldsymbol{\varepsilon}_{\ell}^{p}\right)^{T}\left(\delta \mathbf{x}_{\ell}-\boldsymbol{\varepsilon}_{\ell}^{p}\right)\right] \\
& =E\left[\left(\delta \mathbf{x}_{\ell}\right)^{T}\left(\delta \mathbf{x}_{\ell}\right)\right]-2 E\left[\left(\delta \mathbf{x}_{\ell}\right)^{T} \boldsymbol{\varepsilon}_{\ell}^{p}\right]+E\left[\left(\boldsymbol{\varepsilon}_{\ell}^{p}\right)^{T} \boldsymbol{\varepsilon}_{\ell}^{p}\right] \\
& =E\left[\left|\delta \mathbf{x}_{\ell}\right|^{2}\right]-2 E\left[\left(\delta \mathbf{x}_{\ell}\right)^{T} \boldsymbol{\varepsilon}_{\ell}^{p}\right]+E\left[\left|\boldsymbol{\varepsilon}_{\ell}^{p}\right|^{2}\right]
\end{aligned}
$$

The second term of the last part of Eq. (3.15) is twice the covariance between $\delta \mathbf{x}_{\ell}$ and $\boldsymbol{\varepsilon}_{\ell}^{p}$. For a properly chosen, high quality verification data set, this correlation can be assumed 
to be zero. The third term of the last part of Eq. (3.15) is the variance of the error in the verifying data. Except for the shortest forecast times, this term can be considered small compared to the first term. Hence, Eq. (3.13) can be written as

$$
E\left[v s_{\ell}\right] \approx E\left[t v_{\ell}\right]
$$

where $t v_{\ell}=\left|\delta \mathbf{x}_{\ell}\right|^{2}$. Introducing the notations $V S_{\ell}=E\left[v s_{\ell}\right]$ and $T V_{\ell}=E\left[t v_{\ell}\right]$, Eq. (3.16) can be written in the equivalent form

$$
V S_{\ell}=T V_{\ell}
$$

where $V S_{\ell}$ represents the variance in the ensemble and $T V_{\ell}$ represents the forecast uncertainty at location $\ell$. The two sides of Eq. (3.17) can be estimated by computing averages of $v s_{\ell}$ and $t v_{\ell}$ for a sufficiently large sample of ensemble forecasts and verification data. These estimates can be averaged over the locations to obtain two scalar quantities, VS and $T V$, for comparison. The relation $V S \approx T V$ is often referred to as the spread-skill relationship, because $V S$ characterizes the spatiotemporal mean of the ensemble spread in state space, while $T V$ can be considered an estimate of the (spatiotemporal) mean-square error (skill) of the deterministic forecasts based on the ensemble mean.

\subsubsection{Covariance}

The ensemble perturbations $\mathbf{X}_{\ell}^{k}, k=1,2, \ldots, K$, in a linear sense, span a linear vector space $\mathbb{S}_{\ell}$ for each location $\ell$. The ensemble captures the uncertain forecast features in the local neighborhood $V_{\ell}$ of $\ell$, if the magnitude $\left|\boldsymbol{\varepsilon}_{\ell}^{r(\|)}\right|$ of the projection $\boldsymbol{\varepsilon}_{\ell}^{r(\|)}$ of $\boldsymbol{\varepsilon}_{\ell}^{r}$ onto $\mathbb{S}_{\ell}$ is equal to the magnitude $\left|\boldsymbol{\varepsilon}_{\ell}^{r}\right|$ of $\boldsymbol{\varepsilon}_{\ell}^{r}$. Because the ensemble typically captures only part of the 
forecast uncertainty,

$$
e v_{\ell}=\frac{\left|\boldsymbol{\varepsilon}_{\ell}^{r(\|)}\right|^{2}}{\left|\boldsymbol{\varepsilon}_{\ell}^{r}\right|^{2}} \leq 1 .
$$

We call the ratio $e v$ the explained variance. Under the assumption that the error $\boldsymbol{\varepsilon}_{\ell}^{p}$ in the verifying data has no projection on $\mathbb{S}_{\ell}$, and the contribution of $\boldsymbol{\varepsilon}_{\ell}^{p}$ to $\left|\boldsymbol{\varepsilon}_{\ell}^{r}\right|$ is negligible, the explained variance can be estimated by

$$
e v_{\ell}=\frac{t v s_{\ell}}{t v_{\ell}} \leq 1
$$

where $t v s_{\ell}=\left|\delta \mathbf{x}_{\ell}^{(\|)}\right|^{2}$ and $\delta \mathbf{x}_{\ell}^{(\|)}$is the projection of $\delta \mathbf{x}_{\ell}$ onto $\mathbb{S}_{\ell}$. The smaller the estimated value of $e v_{\ell}$, the lower the efficiency of the ensemble in capturing the forecast uncertainty. Unlike the diagnostic relations discussed earlier, this relationship can be verified for a particular forecast and location. We will take advantage of this property of $e v_{\ell}$ in Sec. 4.

In practice, $\delta \mathbf{x}_{\ell}^{(\|)}$can be computed by projecting $\delta \mathbf{x}_{\ell}$ onto the set of normalized eigenvectors $\left\{\mathbf{u}_{\ell}^{k}: k=1, \ldots, K-1\right\}$ associated with the largest $K-1$ eigenvalues of $\mathbf{P}_{\ell}$, which provide a convenient orthonormal basis for the computations. The mean magnitude $E\left[t v s_{\ell}\right]$ of $\delta \mathbf{x}_{\ell}^{(\|)}$can be estimated by averaging over a sample of forecasts. The resulting local estimates $T V S_{\ell}$, can be further averaged over the locations to obtain a single scalar measure TVS for the characterization of the mean projection. This scalar measure always satisfies the relation $T V S \leq T V$. The larger the difference between $T V S$ and $T V$, the poorer the performance of the ensemble in capturing the spatial structure of the forecast uncertainty. 


\subsection{Results}

\subsubsection{Comparison of $V S, T V$, and $T V S$}

We compute $V S_{\ell}, T V_{\ell}$, and $T V S_{\ell}$ by averaging $v s_{\ell}, t v_{\ell}$, and $t v s_{\ell}$ over all forecasts for January and February at $0000 \mathrm{UTC}$ and $1200 \mathrm{UTC}$, and $V S, T V$, and $T V S$ by averaging $V S_{\ell}, T V_{\ell}$, and $T V S_{\ell}$ over the Northern Hemisphere extratropics $\left(30^{\circ} \mathrm{N}-75^{\circ} \mathrm{N}\right)$. We display the values of all diagnostics, including those of the bias, as functions of forecast time. It is important to note when comparing the evolution of the diagnostics for the ensembles of the different centers that the maximum forecast lead time is not the same for all of them.

The evolution of $V S, T V$, and $T V S$ for ECMWF is displayed in the left panel of Fig. A.1. $V S$ and $T V S$ are much smaller than $T V$ at analysis time. While $V S$ increases rapidly and approaches $T V$ within 48-72 hours, $T V S$ increases more slowly, and asymptotes to $T V$ and $V S$ by about 96 hours. As expected, $T V S<T V$ for all lead times, indicating that the ensemble misses some patterns of forecast uncertainty. However, $T V S$ is only slightly smaller than $T V$ after about 144 hours, and maintains this behavior throughout the remaining lead times. While $V S \approx T V S$ at analysis time, initially $V S$ grows faster than $T V S$, which leads to an overestimation of the magnitude of the part of the forecast uncertainty that is captured by the ensemble. In other words, the successfully captured patterns of uncertainty are inflated in an attempt to better represent the overall magnitude of the forecast uncertainty. Overall, the results for ECMWF are very similar to those of Herrera et al. (2016).

The results for NCEP are shown in the right panel of Fig. A.1. For this ensemble, 
$T V S$ is visibly smaller than $T V$ at all lead times. The main differences in the behavior of the three diagnostics between the NCEP and ECMWF ensembles are that the agreement between the evolutions of $V S, T V$, and $T V S$ is weaker and the convergence of $V S$ to $T V$ is slower, for the NCEP ensemble. In general, the three diagnostics suggest that the performance of the NCEP ensemble is poorer than that of the ECMWF ensemble. Because Herrera et al. (2016) reported similar differences in the performance of the two ensembles, we conclude that ECMWF has managed to maintain its advantage over NCEP.

The results on the evolution of $V S, T V$, and $T V S$ for the remaining TIGGE ensembles are shown in Fig. A.2. The differences between the behaviors of the different ensemble systems are the largest at analysis time. These differences primarily reflect the substantial differences between the techniques for the generation of the initial condition perturbations. The CMC ensemble behaves similarly to the NCEP ensemble, in that $V S$ asymptotes to $T V$ quickly but $T V S$ remains smaller than $T V$ at all forecast times. The CMC and UKMO ensembles overestimate the magnitude of the uncertainty captured by the ensemble by a larger margin at the longer lead times than even the NCEP ensemble. JMA behaves differently, especially at analysis time, at which $V S$ underestimates $T V S$. Because of the more rapid initial growth of $V S, V S$ catches up with $T V S$ by the 12-hour forecast time and remains larger until about the 144-hour forecast time. This is a feature of the JMA ensemble that was also observed by Herrera et al. (2016) and can be attributed to the use of right singular vectors as initial condition perturbations.

The evolution of $V S$ for the KMA ensemble is unique in that it is larger than $T V$ 
until about the 48-hour forecast time. Despite this overinflation of $V S$ at the early forecast times, $V S$ underestimates $T V$ beyond the 96-hour forecast time. In contrast, for the UKMO ensemble, $V S$ matches $T V$ well at all forecast times. In that ensemble, however, $T V S$ significantly underestimates $T V$ at all forecast times. The large discrepancy between $V S$ and $T V S$ shows that the UKMO ensemble fails to capture some important features of uncertainty and it compensates for this deficiency by overinflating the magnitude of the captured part of the uncertainty. Because Herrera et al. (2016) did not observe a similar behavior for the UKMO ensemble, our results indicate that the change implemented at UKMO greatly degraded the performance of the ensemble in this respect.

\subsubsection{The evolution of $M^{2}$}

Figures A.1 and A.2 show a slow but steady growth of the bias, $M^{2}$, as forecast time increases, for all ensembles. Herrera et al. (2016) found that a similar growth of the bias in 2012 was due to growing errors in the prediction of the low-frequency transients. The similarity between the shapes of the curves of $M^{2}$ in Figs. A.1 and A.2 of the present study and Figure 3 of Herrera et al. (2016) suggests that the growth of the bias in 2015 was also due to growing errors in the prediction of the low frequency transients. Fig. A.3, which shows the spatial distribution of $M_{\ell}$ for the ECMWF ensemble, provides strong support to our conjecture. ${ }^{1}$ It shows that similar to the situation in 2012, the locations of the local maxima of $M_{\ell}$ coincide with the locations of the local maxima of the amplitude of the large-scale waves. In addition, these local maxima of the bias occur, because the ensemble

\footnotetext{
${ }^{1}$ Fig. A.3 can be directly compared to Figure 10 of Herrera et al. (2016).
} 
predicts an overly zonal flow at the long lead times (Fig. A.4). An additional similarity with the results for 2012 is that the aforementioned behavior is shown by not only the ECMWF, but all the other ensembles, as well (Fig. A.5).

While the qualitative behavior of $M^{2}$ is very similar between 2012 and 2015, there is an important quantitative difference between the results of the two years: at long lead times, the values of $M^{2}$ are lower in 2015 than in 2012 (about $588 \mathrm{~J} / \mathrm{kg}$ vs. $683 \mathrm{~J} / \mathrm{kg}$ for ECMWF and $650 \mathrm{~J} / \mathrm{kg}$ vs. $847 \mathrm{~J} / \mathrm{kg}$ for NCEP at the 360 -hour lead time). The CMC ensemble, the only other ensemble that provides forecasts for the 360-hour lead time, shows a similar behavior ( $M^{2}$ was reduced from $803 \mathrm{~J} / \mathrm{kg}$ to $599 \mathrm{~J} / \mathrm{kg}$ ). One possibility is that the reduction of $M^{2}$ is due to the progress made by the centers between 2012 and 2015, while another is that it is due to differences between the flow regimes of the two years. To confirm or rule out the latter possibility, next, we compare the zonal anomalies of the time-mean flow for the two years (Fig. A.6).

The region where $M_{\ell}$ is the largest coincides with the locations of strong positive zonal anomalies in both years: in 2012, it is in the north Atlantic region (Herrera et al., 2016), while in 2015, it is in the northeast Pacific region (bottom right panel of Fig. A.3). This observation suggests that, because the ensemble forecasts have difficulties with maintaining the large scale ridges, the systematic error in the long-term ensemble mean forecasts is the largest where a strong ridge is located. This relationship between the location of the local maximum of $M_{\ell}$ and the location of large zonal anomalies suggests that the difference between the flow regimes of 2012 and 2015 do contribute to the differences between the 
values of $M^{2}$ in the long forecast range. The fact that $M^{2}$ is the lowest for the ECMWF ensemble and the highest for the NCEP ensemble in both years suggests that differences between the quality of the ensemble systems also play a role in determining the value of $M^{2}$. However, the differences in the values of $M^{2}$ for the ensembles of the different centers are small compared to the reduction that could be potentially achieved at any of the centers by maintaining the large scale zonal anomalies in the long forecast range. 


\section{EVALUATION OF THE PREDICTIVE SCHEMES}

The ensemble dimension (E-dimension) is a measure of the steepness of the eigenvalue spectrum of $\mathbf{P}_{\ell}$ : the smaller the E-dimension the steeper the spectrum. Satterfield and Szunyogh (2010) found the E-dimension to be a good linear predictor of the lower bound of the explained variance. In addition, Satterfield and Szunyogh (2010) found a strong linear relationship between the ensemble spread and the 95th percentile value of the local forecast error. We show that the aforementioned two linear relationships also hold for the ECMWF ensemble.

\subsection{The predictive linear relations}

\subsubsection{The lower bound of the explained variance}

The E-dimension (Patil et al., 2005; Oczkowski et al., 2005) is

$$
E_{\ell}=\frac{\left(\sum_{k=1}^{K} \sqrt{\lambda_{\ell}^{k}}\right)^{2}}{\sum_{k=1}^{K} \lambda_{\ell}^{k}},
$$

where $\lambda_{\ell}^{1} \geq \lambda_{\ell}^{2} \geq \cdots \geq \lambda_{\ell}^{K} \geq 0$ are the eigenvalues of $\mathbf{P}_{\ell}$. The subscript $\ell$ in the notation $E_{\ell}$ indicates that the E-dimension is computed for local volumes. It takes its smallest possible value of 1 , when the ensemble variance is associated with a single pattern of uncertainty (eigenvector), and its largest possible value of $K$, when the ensemble variance is evenly distributed between $K$ different patterns of forecast uncertainty. Satterfield and Szunyogh (2010) found that the minimum value $m_{\ell}$ of the explained variance $e v_{\ell}$ that the ensemble 
was guaranteed to capture satisfied, to a good approximation, the linear relationship

$$
m_{\ell}=a E_{\ell}+b,
$$

where $a$ and $b$ were empirical scalars determined from a sample of ensemble and verification data.

\subsubsection{The 95th percentile value of the forecast error}

According to Eq. (3.12), the spatiotemporally varying ensemble spread (standard deviation) $v s_{\ell}^{1 / 2}$ is a predictor of the root-mean-square of the forecast uncertainty. Hence, the larger the ensemble spread, the larger the expected magnitude of the forecast uncertainty. What limits the practical quantitative forecast value of the ensemble spread is that for large values of the spread, the magnitude of the forecast error can vary within a wide range (e.g., Figure 4 of Satterfield and Szunyogh, 2011). Satterfield and Szunyogh (2011) found a potentially more useful quantitative relationship between the ensemble spread and the magnitude of the forecast error by noticing that, to a good approximation, the exceptionally large values of the magnitude of the forecast error depended linearly on the ensemble spread. More precisely, they found the relationship

$$
t v_{95}=\left(c \times v s_{\ell}\right)+d,
$$

where $t v_{95}$ was the 95 th percentile value of $t v_{\ell}=|\delta \mathbf{x}|^{2}$ given $v s_{\ell}$. The parameters $c$ and $d$ were empirical scalars determined from a sample of ensemble and verification data. If Eq. (4.3) held for the operational ensembles, it would provide a practical formula for the quantitative prediction of a worst case scenario for the magnitude of the forecast error. 


\subsection{Results}

The explained variance, $e v_{\ell}$, which was defined by Eq. (3.18), determines the efficiency of the ensemble in capturing the patterns of forecast uncertainty. The spatial distribution of the explained variance $e v_{\ell}$ for the ECMWF ensemble averaged over all of the forecasts is displayed in Fig. A.7. As expected based on the result of the earlier studies, the explained variance initially grows rapidly as forecast time increases in the Northern Hemisphere storm track regions (upper right and lower left panels of Fig. A.7). Then, the explained variance continues to grow slowly at all locations, reaching values between 0.75 and 1 by the 360 -hour lead time.

Kuhl et al. (2007) and Satterfield and Szunyogh (2010) observed a negative correlation between $E_{\ell}$ and $e v_{\ell}$ : a lower E-dimension indicated a higher explained variance, especially at forecast times longer than three days. In other words, when and where the ensemble spread was dominated by very few patterns, those patterns provided an efficient representation of the structure of the forecast uncertainty.

As done in the previous studies, we prepare estimates of the joint probability distribution function (JPDF) of the explained variance and the E-dimension. To obtain estimates of the JPDF, we compute relative frequencies of the values of the explained variance and E-dimension for discrete bins of the values for all locations $\ell$ in the $\mathrm{NH}$ extratropics for all forecasts. We use $200 \times 200$ bins by having an increment of 0.25 for E-dimension and 0.005 for the explained variance. The number of occurrences in each bin is normalized by $\Delta E \times \Delta E V \times n$, where $n=305856$ is the total number of data points. Figure A.8 shows 
the resulting estimates of the JPDF at analysis time, and 72-hour, 120-hour, and 360-hour forecast lead times. At analysis time, the maximum value of the E-dimension is close to 25. This is due to ECMWF generating their 50 ensemble perturbations in pairs of negative and positive perturbations, thus half of the perturbations are linearly dependent on the other half. In the first 72 hours, both the E-dimension and the explained variance tend to grow. While the explained variance keeps growing as forecast time increases, albeit at a slower rate than in the first 72 hours, the largest values of the E-dimension decrease (e.g., compare the two right panels of Fig. A.8). The distributions at the 72-hour and the 120-hour lead times 'lean backward', indicating a negative correlation between the two variables, as was expected based on the results of Kuhl et al. (2007) and Satterfield and Szunyogh (2010).

To quantify the relationship between the explained variance and the E-dimension, we fit a function of the form of Eq. (4.2) to the data pairs for each lead time. For the function fitting, we divide the data pairs randomly into training data sets and test data sets. Seventyfive percent of the data points are assigned to the training data set and the remaining twentyfive percent is assigned to the test data set. The data are ordered by values of E-dimension and divided into 100 bins of equal number of data separately for the training and the test sets. For each bin, we calculate the mean of the E-dimension and the minimum value of the explained variance and perform a linear regression on the E-dimension and the explained variance values from the training data set. The linear regression provides the estimates of the parameters $a$ and $b$. We use these values of $a$ and $b$ to predict the minimum of the explained variance in the test data set based on the corresponding values of E-dimension. 
The correlation values $\left(R^{2}\right)$ between the minimum of the explained variance and the mean E-dimension are calculated for the training and test sets for each forecast lead time, denoted by $R^{2}$ training and $R^{2}$ test, respectively. This entire process, beginning from randomizing the data, is repeated 100 times with varying training and test periods to provide a robust analysis. The $R^{2}$ values for each iteration are averaged together.

The $R^{2}$ values are listed in the first two rows in Table B.2. These correlation values represent the average over the 100 iterations, but the graphical illustration of the results in Fig. A.9 shows the results for only one random iteration. In this figure, the dark triangles represent the training data and the open circles represent the test data. The correlation values at analysis time and 360-hour forecast time are low, compared to the values at the 72-hour and 120-hour lead times.

Satterfield and Szunyogh (2010) speculated that outliers were the likely cause of the overprediction of the minimum explained variance values in the test data set. This motivates us to investigate the correlation between the E-dimension and the 5 th percentile value of the explained variance, rather than the minimum. The results are shown in Fig. A.10, with the corresponding average $R^{2}$ values also listed in Table B.2. The correlation between the E-dimension and the 5th percentile of explained variance is much higher in both the training and the test periods than between the E-dimension and the minimum of the explained variance.

Next, we investigate whether or not Eq. (4.3) holds for the ECMWF ensemble. The training and test data sets are constructed similarly to that already described for the es- 
timation of the parameters $a$ and $b$ of Eq. (4.2). The available data is divided into 100 bins of equal number of $v s_{\ell}^{1 / 2}$ values and a linear regression is performed for each forecast lead time. This process is repeated for 100 randomly selected data pairs and the results are shown in the last two rows of Table B.2 and in Fig. A.11. The correlations are high at all forecast lead times, with the highest value of 0.98 for the test data at 72 -hour and 120-hour lead times, indicating that $v s_{\ell}$ is a predictor of the 95 th percentile value of the forecast error. 


\section{CONCLUSIONS}

The two main goals of this study were to update the results of Herrera et al. (2016) on the performance of the operational global ensemble forecast systems in the NH extratropics based on data from January and February of 2015; and to validate two predictive linear relations found by Satterfield and Szunyogh $(2010,2011)$ for operational ensemble forecast data.

Our main conclusions regarding the performance of the ensemble forecast systems are the following:

- The main characteristics of the systems of the different centers, in terms of the efficiency in representing the spatiotemporal evolution of the forecast uncertainty, have not changed much in the last three years. The only exception is the UKMO ensemble, whose performance improved in predicting the magnitude of the forecast uncertainty, and greatly degraded in predicting the patterns of forecast uncertainty. This ensemble was redesigned recently, as it is no longer used to support mediumrange forecasting. It's sole purpose is to provide boundary and initial conditions for short-term limited area ensemble forecasts.

- The ECMWF ensemble continues to provide the highest quality forecasts, with respect to the performance measures of this present study.

- All ensembles have major difficulties with predicting the large scale atmospheric flow in the long forecast range (longer than 10 days). These difficulties are due to the 
inability of the models to maintain the large-scale zonal anomalies of the atmospheric flow in the long forecast range.

Our main conclusion regarding the predictive relations of Satterfield and Szunyogh $(2010,2011)$ is that they hold for the operational ensembles, as well. These two relations could be utilized for the routine operational prediction of (i) the reliability of ensemble forecasts in capturing the local structure of the forecast uncertainty, and (ii) the 95th percentile value of the forecast error. 


\section{REFERENCES}

Bougeault, P., and Coauthors, 2010: The THORPEX Interactive Grand Global Eensemble (TIGGE). Bull. Amer. Meteor. Soc., 91, 1059-1072.

Buizza, R., J. Tribbia, F. Molteni, and T. Palmer, 1993: Computation of optimal unstable structures for a numerical weather prediction model. Tellus, 45, 388-407.

Herrera, M. A., I. Szunyogh, and J. Tribbia, 2016: Forecast uncertainty dynamics in the THORPEX Interactive Grand Global Ensemble (TIGGE). Mon. Wea. Rev., 144, In press.

Kuhl, D., and Coauthors, 2007: Assessing predictability with a local ensemble kalman filter. J. Atmos. Sci., 64, 1116-1140.

Oczkowski, M., I. Szunyogh, and D. J. Patil, 2005: Mechanisms for the development of locally low-dimensional atmospheric dynamics. J. Atmos. Sci., 62, 20 742-2431.

Patil, D. J., B. R. Hunt, E. Kalnay, J. A. Yorke, and E. Ott, 2005: Local low-dimensionality of atmospheric dynamics. Phys. Rev. Lett., 86, 5878-5881.

Satterfield, E., and I. Szunyogh, 2010: Predictability of the performance of an ensemble forecast system: Predictability of the space of uncertainties. Mon. Wea. Rev., 138, 962981.

Satterfield, E., and I. Szunyogh, 2011: Assessing the performance of an ensemble forecast 
system in predicting the magnitude and the spectrum of analysis and forecast uncertainties. Mon. Wea. Rev., 139, 1207-1223.

Swinbank, R., and Coauthors, 2016: The TIGGE project and its achievements. Bull. Amer. Meteor. Soc., 97, 49-67.

Talagrand, O., 1981: A study of the dynamics of four-dimensional data assimilation. Tellus, $33,43-60$. 


\section{APPENDIX A}

\section{FIGURES}
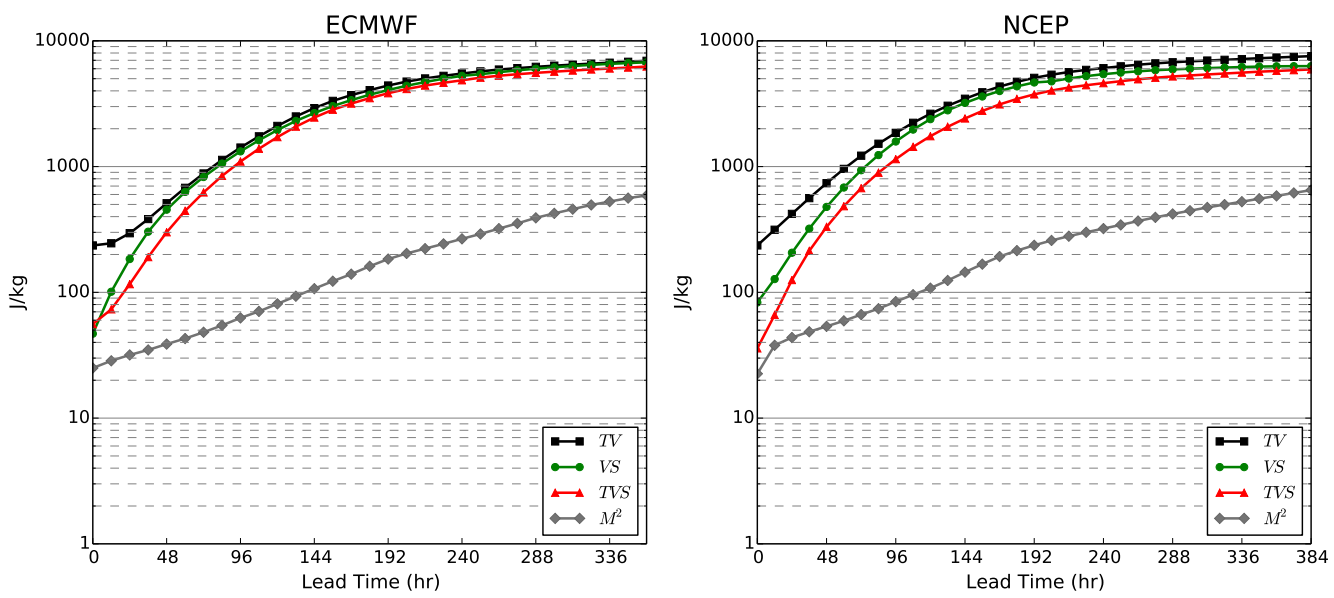

Figure A.1: Spatiotemporal evolution of the diagnostics, $T V, V S, T V S$, and $M^{2}$, for the ECMWF (left) and NCEP (right) ensembles, averaged over the Northern Hemisphere extratropics and all of the available forecasts. 

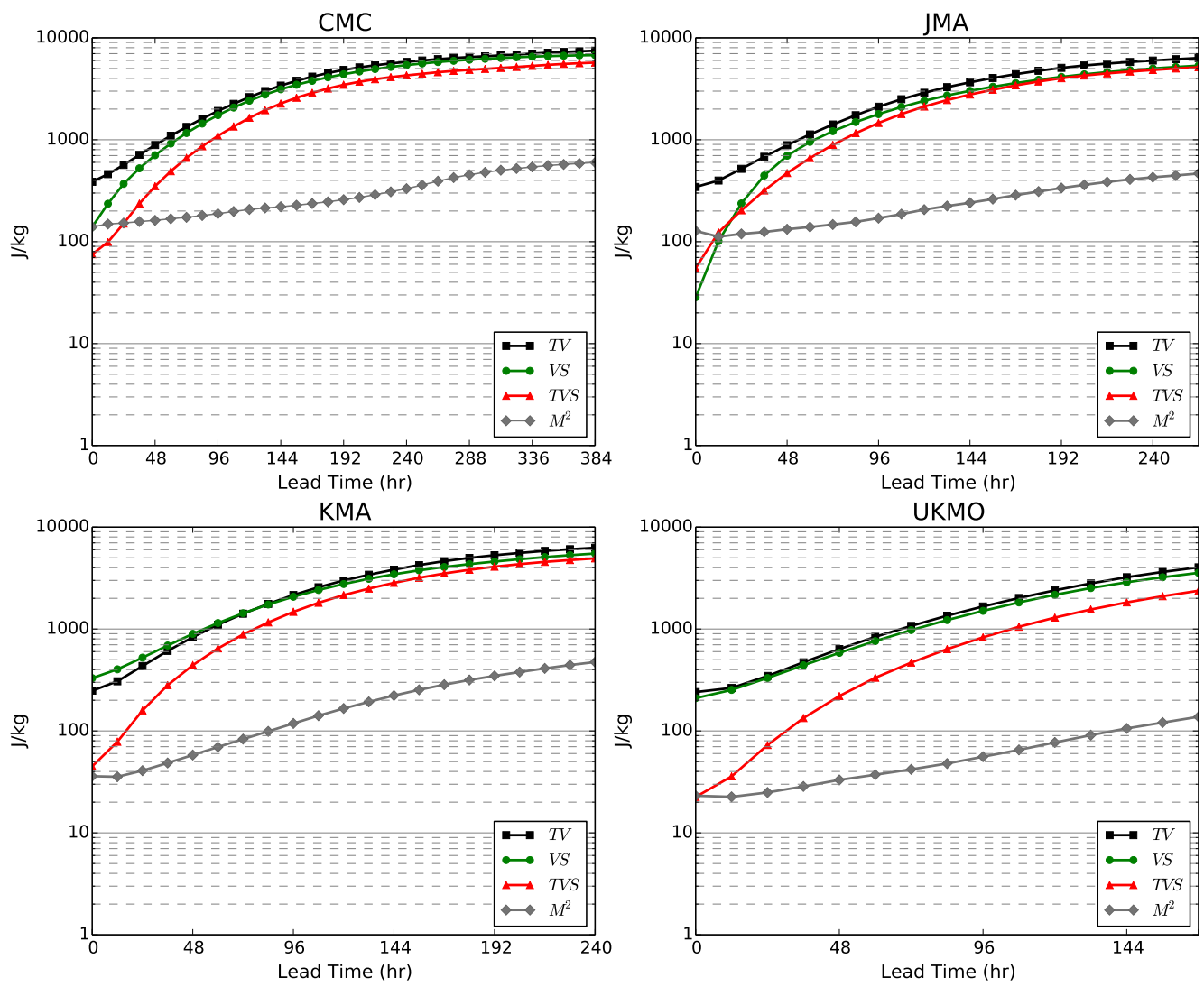

Figure A.2: Spatiotemporal evolution of the diagnostics, $T V, V S, T V S$, and $M^{2}$, for the CMC (top left), JMA (top right), KMA (bottom left), and UKMO (bottom right) ensembles, averaged over the Northern Hemisphere extratropics and all of the available forecasts. The maximum number of lead times vary for these models. 

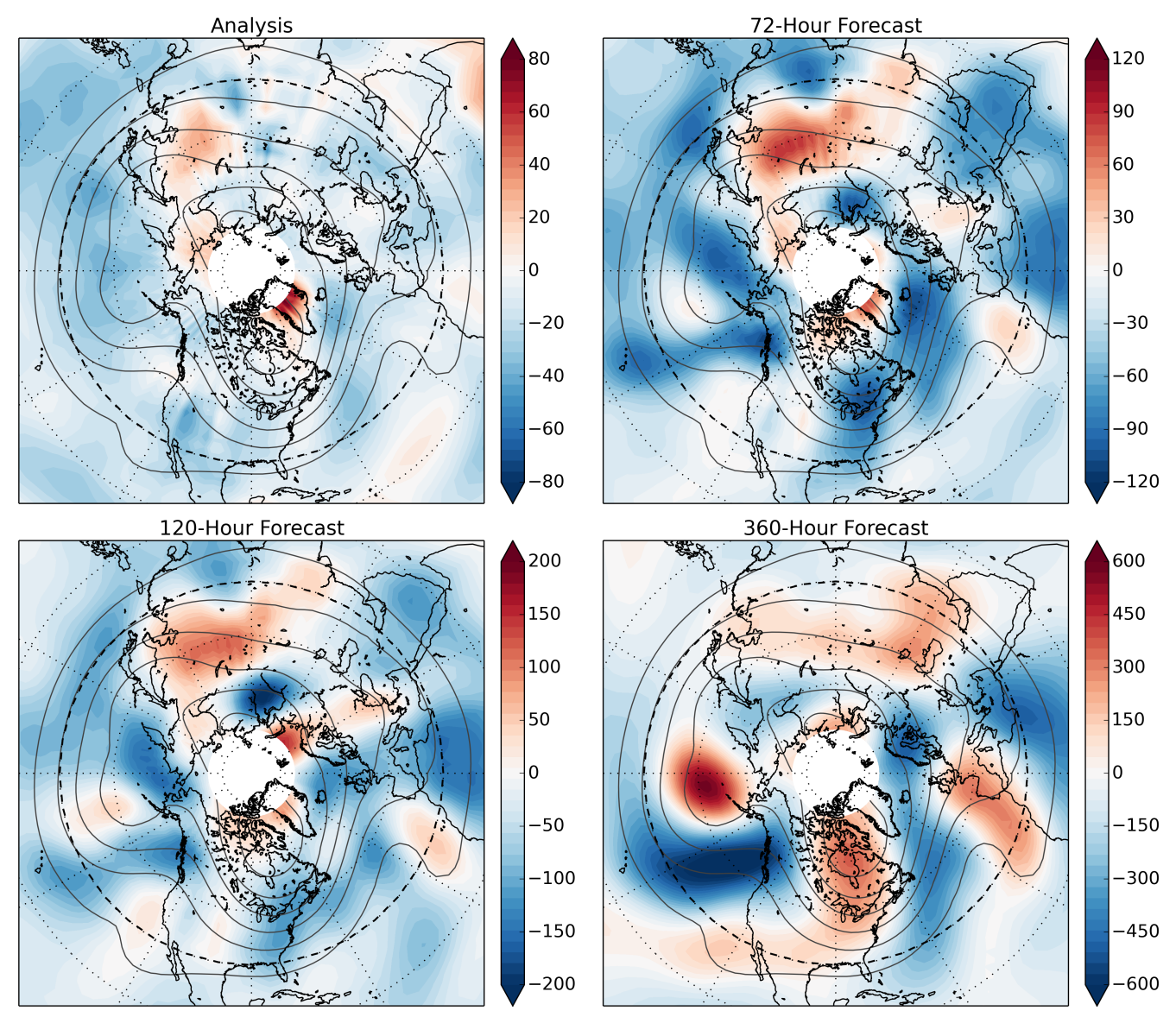

Figure A.3: Spatial distribution of mean bias for the ECMWF ensemble in $(\mathrm{J} / \mathrm{kg})^{1 / 2}$, averaged over all of the forecasts, for analysis (top left), the 72-hour (top right), the 120-hour (bottom left), and the 360-hour (bottom right) forecast lead times, shown as color shades. The black contours represent the mean geopotential height at $500 \mathrm{hPa}$. The heavy dashed line denotes the southern boundary of the verification region $\left(30^{\circ} \mathrm{N}\right)$. 

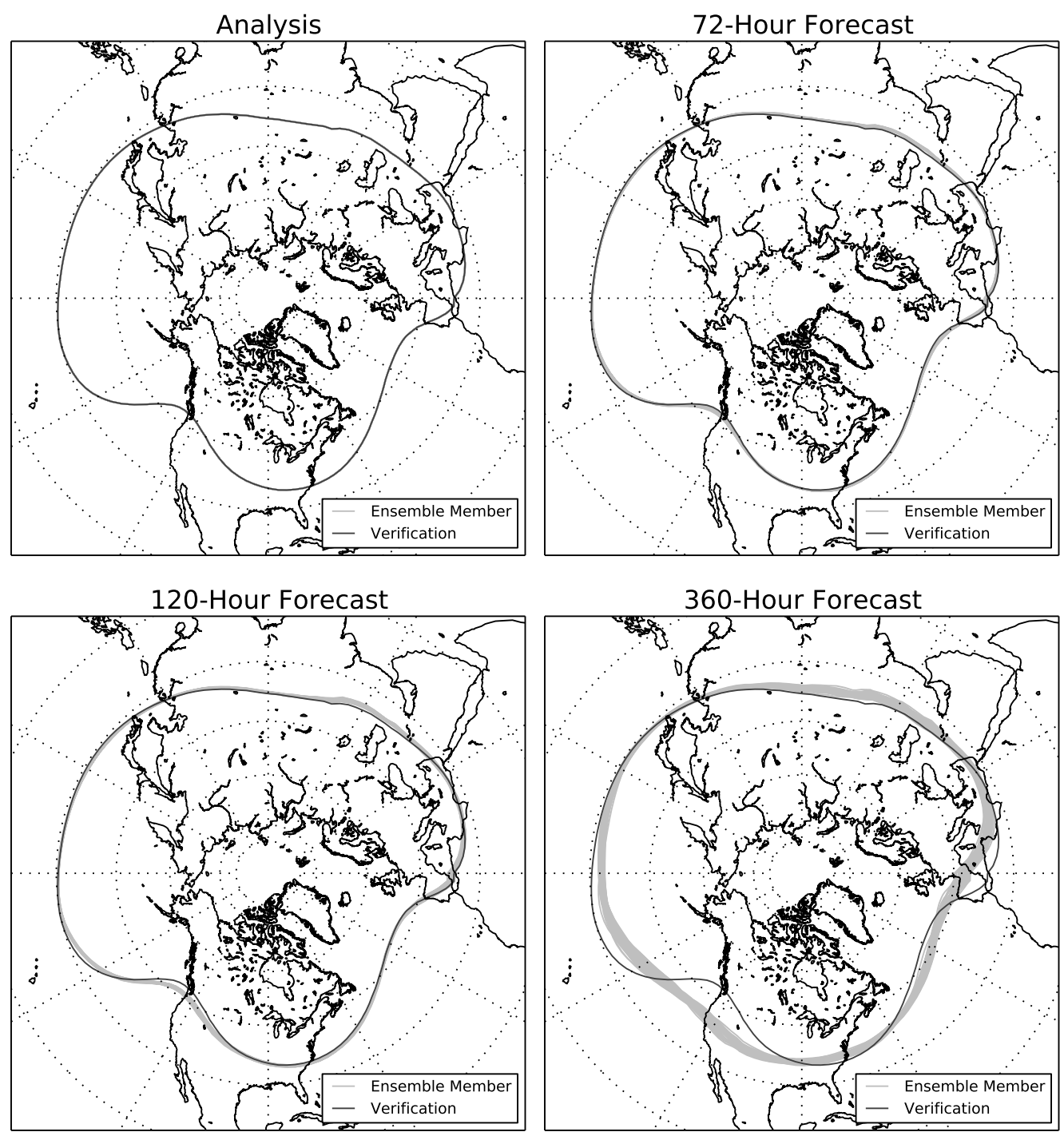

Figure A.4: Spaghetti diagram for the ECMWF ensemble at analysis time (top left), the 72-hour (top right), the 120-hour (bottom left), and the 360-hour (bottom right) forecast lead times, represented by the ensemble members for the averaged forecasts over the time period, in gray, and the mean ECMWF analyses as the verification, in black. The ensemble members and verification are represented as 5625 gpm isohypses. 

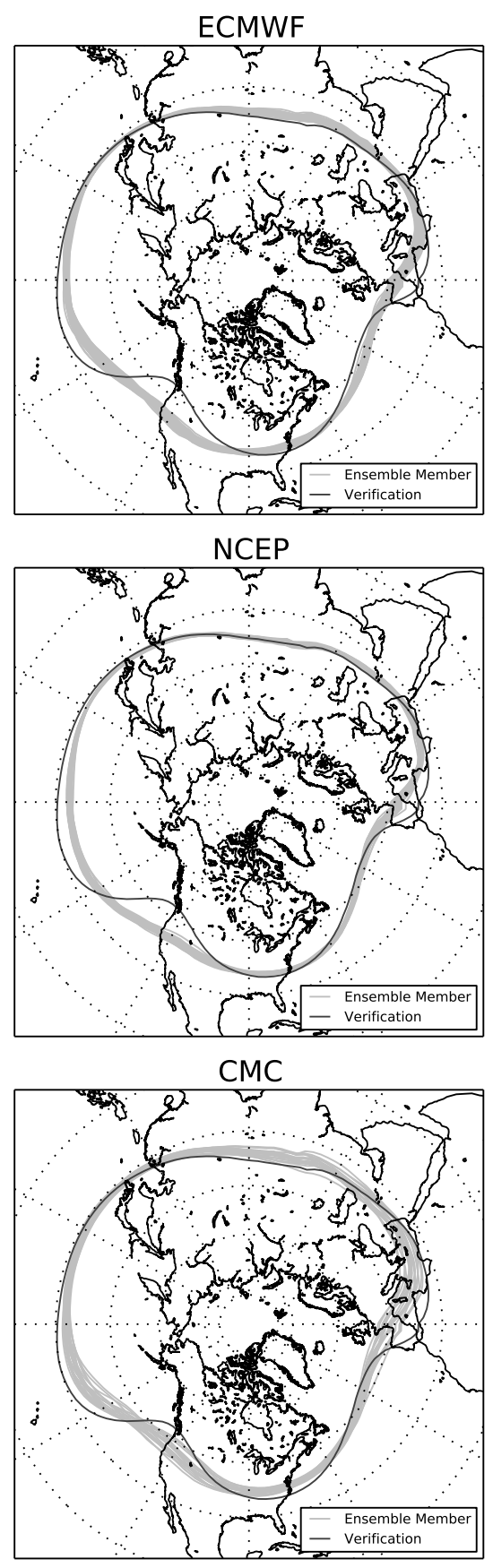

Figure A.5: Spaghetti diagram for the ECMWF (top), NCEP (middle), and CMC (bottom) ensembles at the 360-hour forecast lead time, represented by the ensemble members for the averaged forecasts over the time period, in gray, and the mean ECMWF analyses as the verification, in black. The ensemble members and verification are represented as 5625 gpm isohypses. (Results are not shown for the remaining ensembles, because they do not provide forecasts at the 360 -hour lead time.) 

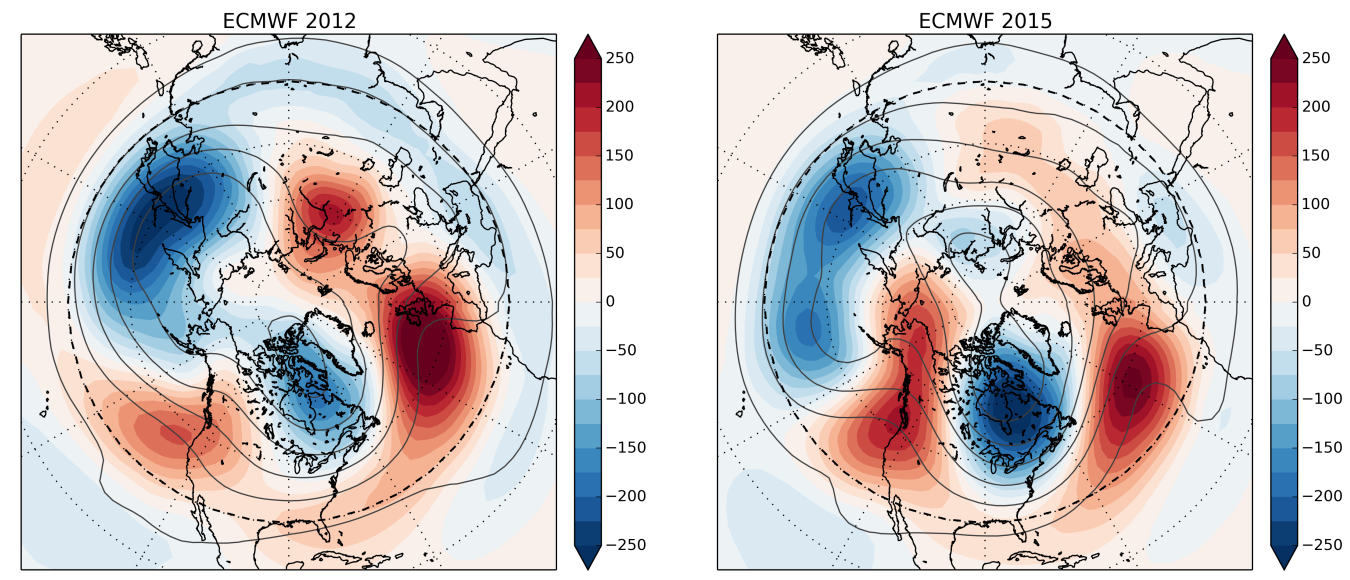

Figure A.6: Zonal anomalies of the time-mean flow for January-February for the ECMWF ensemble from 2012 (left) and from 2015 (right). The color shades represent the zonal anomalies (in geopotential meters) and the black contours represent the mean geopotential height at $500 \mathrm{hPa}$. The heavy dashed line denotes the southern boundary of the verification region $\left(30^{\circ} \mathrm{N}\right)$. 

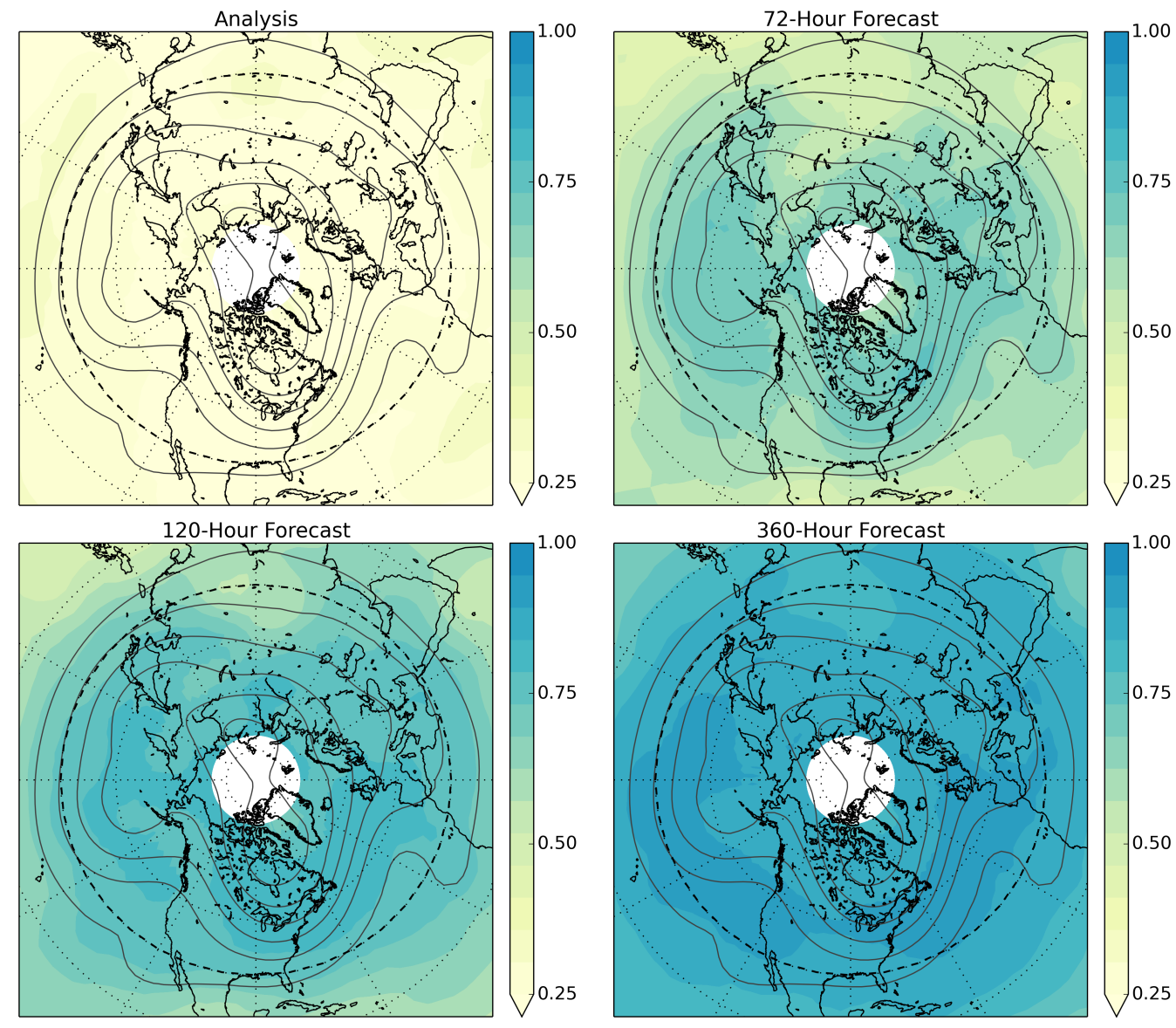

Figure A.7: Spatial distribution of explained variance for the ECMWF ensemble, averaged over all of the forecasts, for analysis (top left), the 72-hour (top right), the 120-hour (bottom left), and 360-hour (bottom right) forecast lead times, shown as the color shades. The black contours represent the mean geopotential height at $500 \mathrm{hPa}$. The heavy dashed line denotes the southern boundary of the verification region $\left(30^{\circ} \mathrm{N}\right)$. 
ECMWF
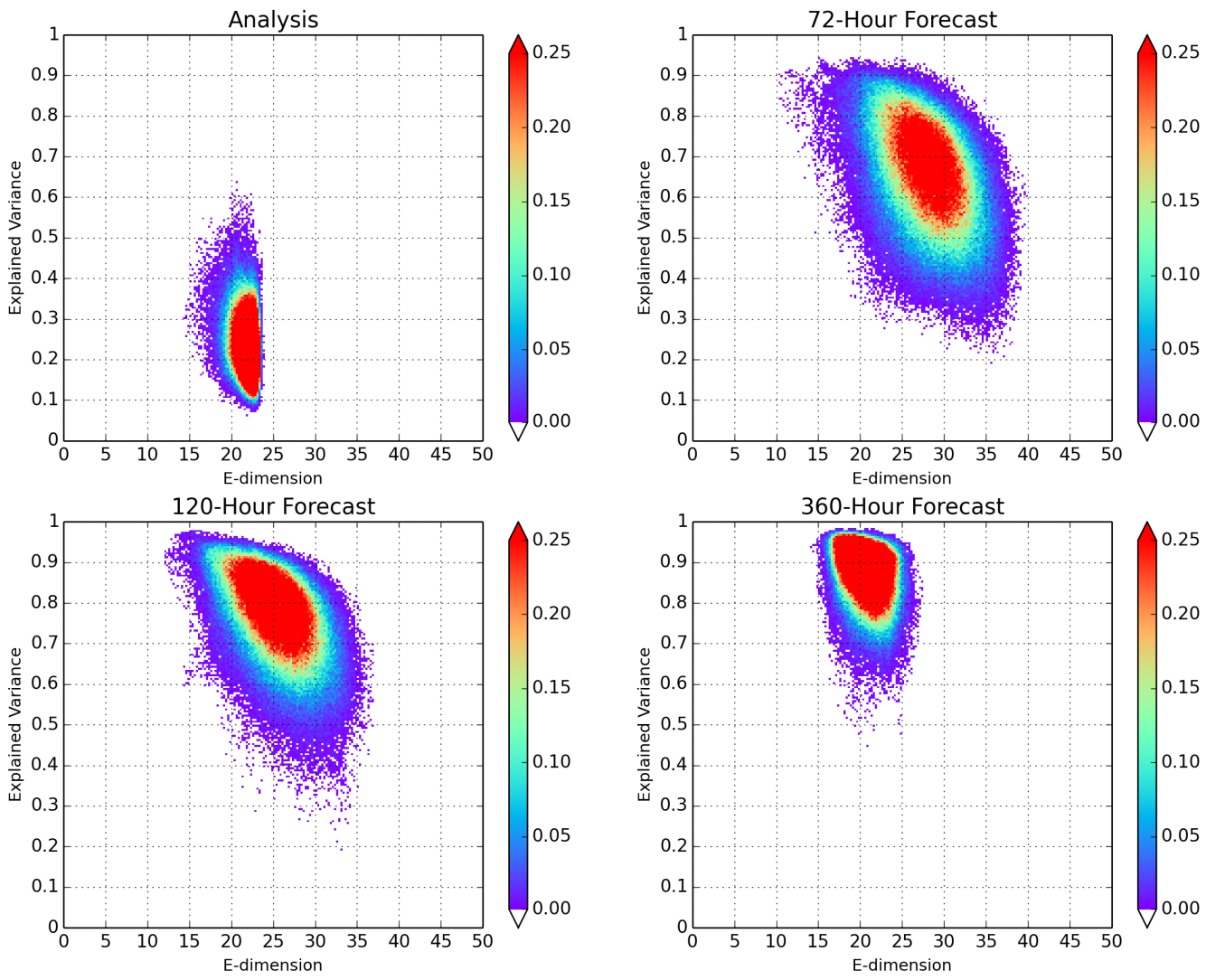

Figure A.8: The joint probability distribution of the E-dimension and the explained variance for the ECMWF ensemble in the Northern Hemisphere extratropics for the analysis (top left), the 72-hour (top right), the 120-hour (bottom left), and the 360-hour (bottom right) forecast lead times. The bin increments are defined as $\Delta E=0.25$ and $\Delta e v=0.005$. The maximum possible value for E-dimension for the ECMWF ensemble is 50. 
ECMWF
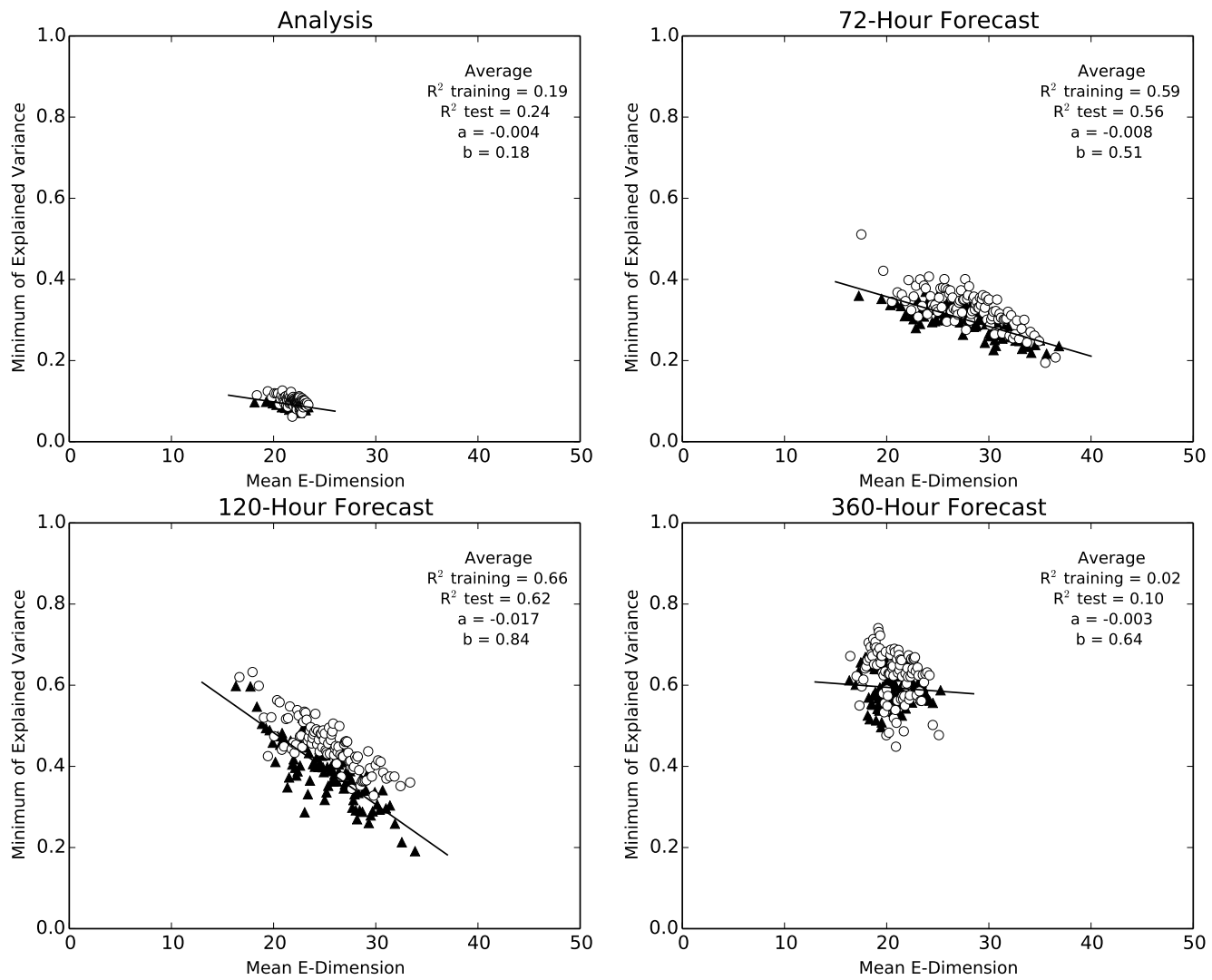

Figure A.9: Mean E-dimension and the minimum of explained variance of data divided equally into 100 bins for the NH extratropics. The training data is represented as triangles, and the linear regression line is fitted to these data. The test data is represented as open circles, and would fall on this line if the linear model was perfect. Shown are the distributions for the analysis time (top left), the 72-hour (top right), the 120-hour (bottom left), and the 360-hour (bottom right) forecast lead times. The legends show the average correlation values between mean E-dimension and minimum explained variance for the training data set $\left(R^{2}\right.$ training) and test data set $\left(R^{2}\right.$ test), as well as the average $a$ and $b$ values. 
ECMWF
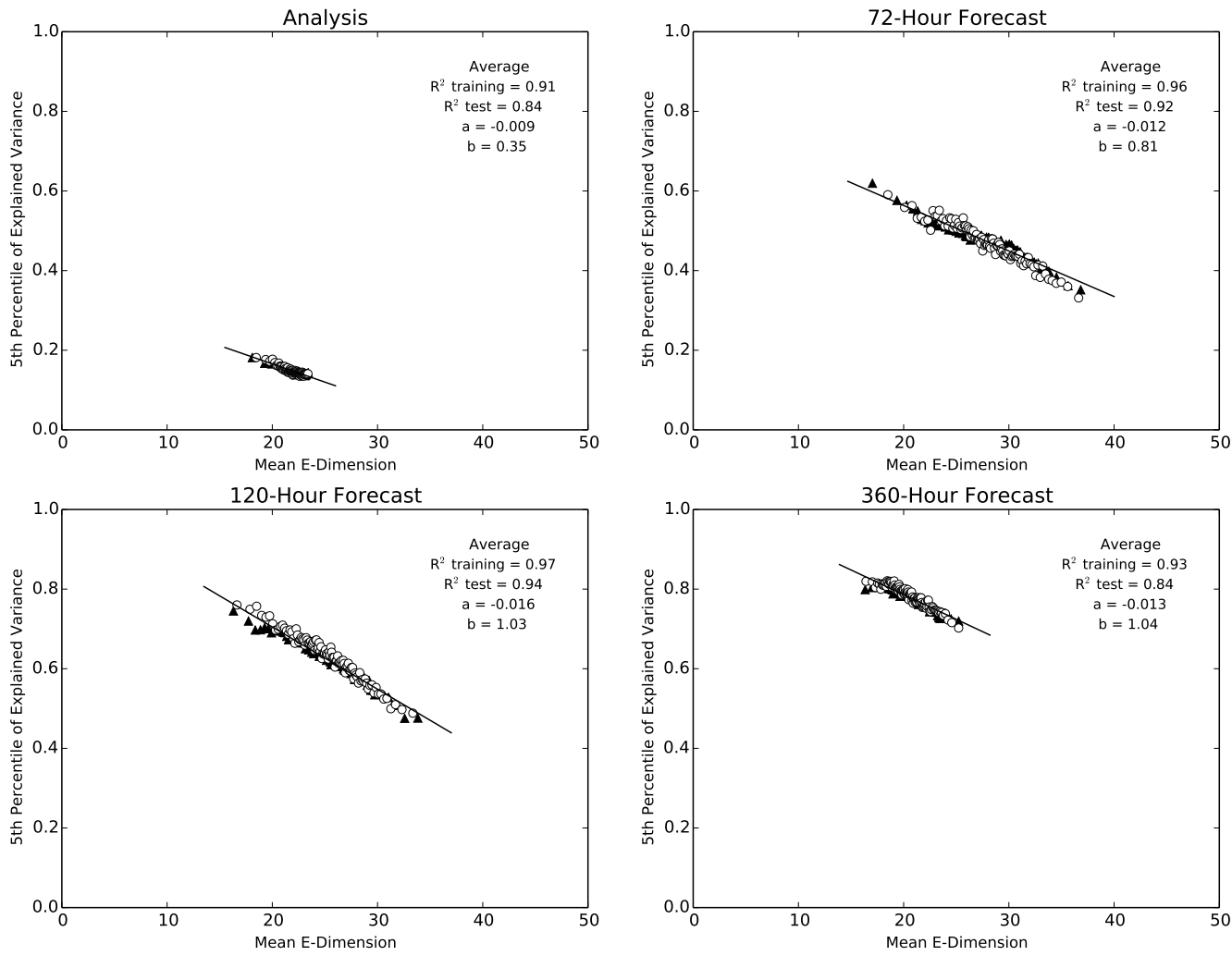

Figure A.10: Mean E-dimension and the 5th percentile of explained variance of data divided equally into 100 bins for the $\mathrm{NH}$ extratropics. The training data is represented as triangles, and the linear regression line is fitted to these data. The test data is represented as open circles, and would fall on this line if the linear model was perfect. Shown are the distributions for the analysis time (top left), the 72-hour (top right), the 120-hour (bottom left), and the 360-hour (bottom right) forecast lead times. The legends show the average correlation values between mean E-dimension and 5th percentile of explained variance for the training data set ( $R^{2}$ training) and test data set ( $R^{2}$ test), as well as the average $a$ and $b$ values. 
ECMWF
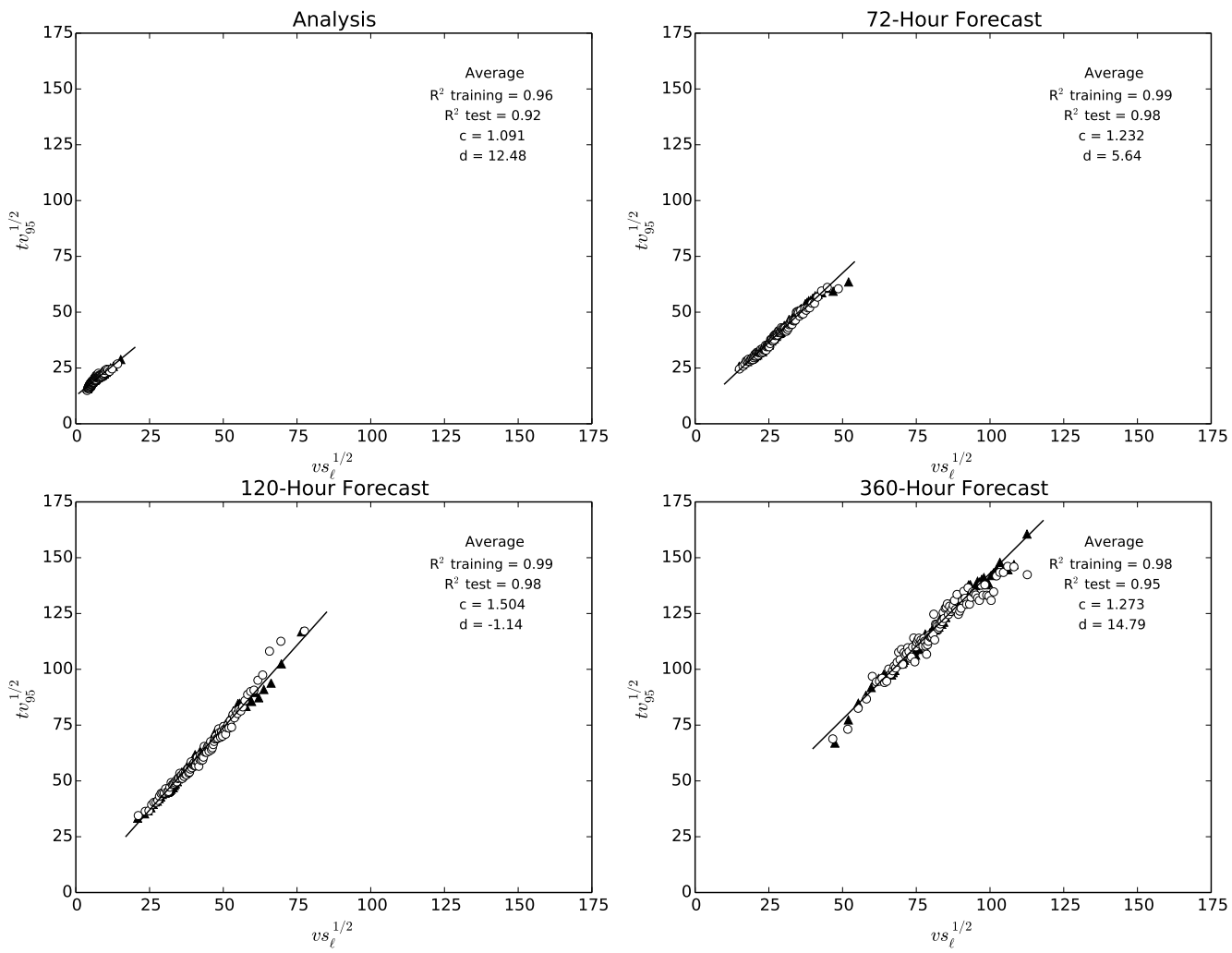

Figure A.11: Mean $v s_{\ell}^{1 / 2}$ and the 95th percentile of $t v_{\ell}^{1 / 2}$ of data divided equally into 100 bins for the $\mathrm{NH}$ extratropics. The training data is represented as triangles, and the linear regression line is fitted to these data. The test data is represented as open circles, and would fall on this line if the linear model was perfect. Shown are the distributions for the analysis time (top left), the 72-hour (top right), the 120-hour (bottom left), and the 360-hour (bottom right) forecast lead times. The legends show the average correlation values between mean $v s_{\ell}^{1 / 2}$ and the 95th percentile of $t v_{\ell}^{1 / 2}$ for the training data set $\left(R^{2}\right.$ training) and test data set ( $R^{2}$ test), as well as the average $c$ and $d$ values. 


\section{APPENDIX B}

\section{TABLES}

Table B.1: Ensemble Forecast Systems

\begin{tabular}{|c|c|c|c|c|}
\hline \hline NWP Center & $\begin{array}{c}\text { Representation of } \\
\text { Model Error and } \\
\text { Uncertainty }\end{array}$ & $\begin{array}{c}\text { Initial Perturbation } \\
\text { Strategy }\end{array}$ & $\begin{array}{c}\text { Max } \\
\text { Forecast } \\
\text { Lead Time }\end{array}$ & $\begin{array}{c}\text { Ensemble } \\
\text { Size }\end{array}$ \\
\hline ECMWF & SKEB/SPPT & $\begin{array}{c}\text { Singular Vectors } \\
\text { and EDA }\end{array}$ & 360 Hours & $\begin{array}{c}50 \\
\text { Members }\end{array}$ \\
\hline NCEP & STTP & $\begin{array}{c}\text { Ensemble } \\
\text { Transform and } \\
\text { Rescaling }\end{array}$ & 384 Hours & $\begin{array}{c}20 \\
\text { Members }\end{array}$ \\
\hline CMC & SPPT/SKEB & EnKF & 384 Hours & $\begin{array}{c}20 \\
\text { Members }\end{array}$ \\
\hline JMA & SPPT & Singular Vectors & 264 Hours & $\begin{array}{c}26 \\
\text { Members }\end{array}$ \\
\hline KMA & SKEB/RP & ETKF & 240 Hours & $\begin{array}{c}23 \\
\text { Members }\end{array}$ \\
\hline UKMO & SKEB/RP & ETKF & 168 Hours & $\begin{array}{c}11 \\
\text { Members }\end{array}$ \\
\hline
\end{tabular}


Table B.2: $R^{2}$ Values for the regressions of Sec. 4

\begin{tabular}{|c|c|c|c|c|c|}
\hline \hline & Analysis & 72 -Hours & 120 -Hours & 360 -Hours \\
\hline $\begin{array}{c}E_{\ell} \text { vs. Minimum } \\
e v_{\ell}\end{array}$ & Training Data & 0.19 & 0.59 & 0.66 & 0.02 \\
\cline { 2 - 6 } & Test Data & 0.24 & 0.56 & 0.62 & 0.10 \\
\hline $\begin{array}{c}E_{\ell} \text { vs. 5th } \\
\text { Percentile } e v_{\ell}\end{array}$ & Training Data & 0.91 & 0.96 & 0.97 & 0.93 \\
\cline { 2 - 6 } & Test Data & 0.84 & 0.92 & 0.94 & 0.84 \\
\hline $\begin{array}{c}\sqrt{v s_{\ell}} \text { vs. 95th } \\
\text { Percentile } \sqrt{t v_{\ell}}\end{array}$ & Training Data & 0.96 & 0.99 & 0.99 & 0.98 \\
\cline { 2 - 6 } & Test Data & 0.92 & 0.98 & 0.98 & 0.95 \\
\hline
\end{tabular}

